\title{
Formation of bamboo-shape carbon nanotubes by controlled rapid decomposition of picric acid
}

\author{
Yi Lu ${ }^{\text {a, c,*, Zhenping Zhu }}{ }^{\text {a, b,*, Dangsheng Su }}{ }^{\mathrm{b}}$, Di Wang ${ }^{\mathrm{b}}$, \\ Zhenyu Liu and Robert Schlögl ${ }^{\mathrm{b}}$ \\ ${ }^{a}$ State Key Laboratory of Coal Conversion, Institute of Coal Chemistry, Chinese Academy of \\ Sciences, Taiyuan 030001, PR China \\ ${ }^{b}$ Department of Inorganic Chemistry, Fritz-Haber-Institute of MPG, Berlin 14195, Germany \\ ${ }^{\mathrm{c}}$ Graduate School of the Chinese Academy of Sciences, Beijing 100039, PR China \\ "Corresponding authors. Tel.: +86 351404 8715; fax: +86 3514041153
}

\begin{abstract}
In the presence of catalysts, carbon nanotubes (CNTs) can efficiently grow in the environment generated by the rapid decomposition of normal explosives. Controlling the reaction parameters of a mixture of picric acid (PA) with cobalt acetate and paraffin can lead to a welldefined morphology of CNTs. The formation of bamboo-shape tubes is favorable at relatively high $\mathrm{Co}(\mathrm{AC})_{2} / \mathrm{PA}$ and paraffin/PA ratios. It is found that the bamboo-shape tubes are different in morphology and structure and can be categorized roughly into two types, according to the participation of the catalyst nanoparticles. The formation of the two types is discussed.
\end{abstract}

Keywords: A. Carbon nanotubes; C. Transmission electron microscopy, X-ray diffraction; D. Microstructure

\section{Introduction}

Recently, carbon nanotubes with special structures such as bamboo-shape [1] and [2], Yjunctions [3], sea urchins [4] and coils [5] have attracted much attention. As the most common member of this family, bamboo-shape CNTs, constructed by many separated hollow compartments, have been frequently investigated to explore their unique structure-associated properties and to understand the relationship between their formation and the growth of normal tubes that exhibit continuous hollow channels. Exploring the appropriate growth conditions of bamboo-shape tubes versus normal tubes would also lead to their controllable syntheses and tune their microstructures selectively.

Until now, bamboo-shape nanotubes have been fabricated through various processes such as arc-discharge of graphite electrodes [1] and [2], pyrolysis of organometallic compounds [6] and [7], thermal chemical vapor deposition (CVD) [8] and [9], and plasma-enhanced CVD 
[10], [11], [12] and [13]. Some corresponding growth models have also been proposed [1], [2], [6] and [8]. Saito et al. [1] and [2] first observed bamboo-shape CNTs in the products from arc discharge evaporation of nickel-loaded graphite and proposed that they are formed from "jumps" of the metal particles out of the graphite sheath to the top of the tube at regular time intervals, driven by the stress accumulated in the graphitic sheath. Kovalevski and Safronov [6] obtained bamboo-shape CNTs through catalytic pyrolysis of acetylene, benzene and ethylene at temperatures between 1950 and $2600{ }^{\circ} \mathrm{C}$ with metal salts (chlorides, sulfides or nitrides of $\mathrm{Fe}, \mathrm{Co}$ and $\mathrm{Ni}$ ) as catalysts. They further proposed a kinetic model for the formation of different types of hollow carbons, in which vapor pressure-driven movement of metal catalyst particles is a key factor for the formation of one-dimension hollow carbon structures and the bamboo-shape CNTs are formed when the rate of catalyst movement is slower than the rate of carbon growth. Lee and Park [8] obtained aligned bamboo-shape CNTs from Fe-catalyzed vapor deposition of acetylene at temperatures ranging from 550 to $950{ }^{\circ} \mathrm{C}$. Based on the observations that the curvature of the compartment layers in the bamboo-like structure are directed to the tube tip and that no catalytic particles are encapsulated in the closed tip, they speculated that the bamboo-shape tubes are formed following a base growth model.

We recently reported that in the presence of Co or Ni catalysts, multi-walled CNTs could well grow in a special high-temperature and high-pressure environment generated from the controlled rapid decomposition (detonation) of common carbon-containing explosives such as $m$-dinitrobenzene [14] and picric acid [15] and [16]. This detonation approach represents a simple, cheap and fast synthetic route towards CNTs without any external energy supply and complicated reaction controlling system. The nanosized catalyst particles are in situ formed from the detonation-induced decomposition and reduction of the precursor compounds without sophisticated preparation procedures. Addition of appropriate amount of hydrocarbons into the reaction system can improve the product yield without influencing the CNT structures. Bamboo-shape tubes were also observed in the products. The yield and structure of the carbon products are determined by the mixture recipe (the catalyst-explosive ratio, and the carbon additive-explosive ratio) and explosive loading density.

In the present paper, we focus on the relationship between the variation of these parameters and the formation processes and give indications towards the preparation of single species of CNTs-bamboo-shape tubes. The morphologies and structures of the bamboo-shape CNTs and the structure of catalyst particles are characterized by transmission electron microscopy (TEM), electron energy-loss spectroscopy (EELS) and X-ray diffraction (XRD). A detailed discussion is given on the growth mechanism of bamboo-shape CNTs.

\section{Experimental}

Picric acid (PA) was used as the explosive to generate the high temperature required and to provide part of carbon species for assembling CNTs. Cobalt acetate $\left(\mathrm{Co}(\mathrm{AC})_{2}\right)$ and liquid paraffin were mixed mechanically in desired ratios (see Table 1), serving as catalyst precursor and additional carbon source, respectively. The experiments were performed in a sealed cylindrical stainless steel pressure vessel (14 $\mathrm{mm}$ in inner diameter, $70 \mathrm{~mm}$ in length) equipped with a pressure gauge. The rapid decomposition of the mixture was induced by external heating $\left(20^{\circ} \mathrm{C} / \mathrm{min}\right)$ by a furnace to $310^{\circ} \mathrm{C}$ and evidenced by a sudden pressure rise (up to $50 \mathrm{MPa}$ dependent on the loading density of PA), which resulted from large amount of gaseous products, mainly $\mathrm{CO}, \mathrm{CO}_{2}$, and $\mathrm{N}_{2}$. After the decomposition, high temperature up to $1000{ }^{\circ} \mathrm{C}$ was generated inside the vessel. The vessel was then cooled in air to ambient temperature and emptied from the gaseous products. The solid products were collected. 
Table 1.

The experimental parameters and their effects on the morphologies of formed carbon nanotubes

\begin{tabular}{|c|c|c|c|c|c|c|}
\hline Sample & $\begin{array}{l}\text { PA loading } \\
\text { density } \\
\left(\mathrm{g} / \mathrm{cm}^{3}\right)\end{array}$ & $\begin{array}{l}\mathrm{Co}(\mathrm{AC})_{2} / \mathrm{PA} \\
\text { mass ratio }\end{array}$ & $\begin{array}{l}\text { Paraffin/PA } \\
\text { mass ratio }\end{array}$ & $\begin{array}{l}\text { Total carbon } \\
\text { density }^{\mathrm{a}} \\
\left(\mathbf{m o l} / \mathbf{c m}^{3}\right)\end{array}$ & $\begin{array}{l}\mathrm{C} / \mathrm{Co} \\
\text { atomic } \\
\text { ratio }\end{array}$ & $C_{\text {bamboo }} / C_{\text {normal }}$ \\
\hline 1 & 0.2 & $1 / 20$ & $1 / 4$ & 0.00823 & 143 & $1 / 20$ \\
\hline 2 & 0.2 & $1 / 20$ & $1 / 3$ & 0.00942 & 164 & $1 / 4$ \\
\hline 3 & 0.2 & $1 / 20$ & $1 / 2$ & 0.01180 & 205 & $3 / 2$ \\
\hline 4 & 0.2 & $1 / 15$ & $1 / 3$ & 0.00949 & 126 & $15 / 1$ \\
\hline 5 & 0.2 & $1 / 10$ & $1 / 3$ & 0.00965 & 84 & $20 / 1$ \\
\hline 6 & 0.2 & $1 / 5$ & $1 / 3$ & 0.01011 & 44 & $15 / 1$ \\
\hline 7 & 0.2 & $1 / 3$ & $1 / 3$ & 0.01071 & 28 & $9 / 1$ \\
\hline 8 & 0.1 & $1 / 15$ & $1 / 3$ & 0.00728 & 97 & $20 / 1$ \\
\hline 9 & 0.05 & $1 / 15$ & $1 / 3$ & 0.00617 & 82 & $25 / 1$ \\
\hline
\end{tabular}

${ }^{\text {a }}$ Calculated from the carbons contained in $\mathrm{PA}, \mathrm{Co}(\mathrm{AC})_{2}$, and paraffin.

${ }^{\mathrm{b}} C_{\mathrm{bamboo}} / C_{\text {normal }}$ is a ratio of the count of bamboo-shape tubes to the count of normal tubes, as estimated from TEM observations.

The solid products were characterized by TEM operating at an accelerating voltage of $75 \mathrm{kV}$, high resolution TEM operating at an accelerating voltage of $200 \mathrm{kV}$, EELS and XRD using $\mathrm{Cu} \mathrm{K} \alpha$ radiation. For TEM analyses, the as-synthesized products were dispersed by ultrasonic treatment for $10 \mathrm{~min}$ in ethanol and then placed on holey carbon films on copper grids.

\section{Results and discussion}

\subsection{Effects of experimental parameters on CNT morphology}

Table 1 presents the experimental parameters and the corresponding ratios of bamboo-shape tubes to normal tubes $\left(C_{\text {bamboo }} / C_{\text {normal }}\right)$ estimated by TEM observations. Since all of the three chemicals involved in the reaction system contain carbon atoms and cooperatively contribute to the formation of the nanotubes, total carbon density and carbon/cobalt atomic ratio are also presented according to the calculation from the experimental parameters employed. As shown in Table 1, the loading density of PA and the recipe of the mixture exhibit significant influences on the morphology of the grown tubes. High PA loading density and low ratios of $\mathrm{Co}(\mathrm{AC})_{2} / \mathrm{PA}$ and paraffin/PA are necessary for sound growth of normal CNTs. Fig. 1a shows a TEM image of the nanotubes produced under such conditions, with PA loading density, $\mathrm{Co}(\mathrm{AC})_{2} / \mathrm{PA}$ and paraffin/PA mass ratios of $0.2 \mathrm{~g} / \mathrm{cm}^{3}, 1 / 20$ and $1 / 4$, respectively [15]. Most of the tubes exhibit continuous hollow channels, although small amount of bamboo-shape tubes (with $C_{\text {bamboo }} / C_{\text {normal }}$ of about $1 / 20$ ) are also observed. The outer diameters and lengths of these normal tubes are in the range of $12-50 \mathrm{~nm}$ and $0.5-40 \mu \mathrm{m}$, respectively. When loading density of PA decreases from 0.2 to $0.05 \mathrm{~g} / \mathrm{cm}^{3}$, the total yield of CNTs decreases sharply from $80 \%$ to $20 \%$ (compared to total carbons) whereas $C_{\text {bamboo }} / C_{\text {normal }}$ ratio seems to have a slight increase (samples 4, 8, 9 in Table 1). These results suggest that the formation of 
bamboo-shape CNTs is associated with relatively low temperatures since the present system is self-heated by the chemical-thermal energy transformation of PA and the loading density of PA determines the system temperature.
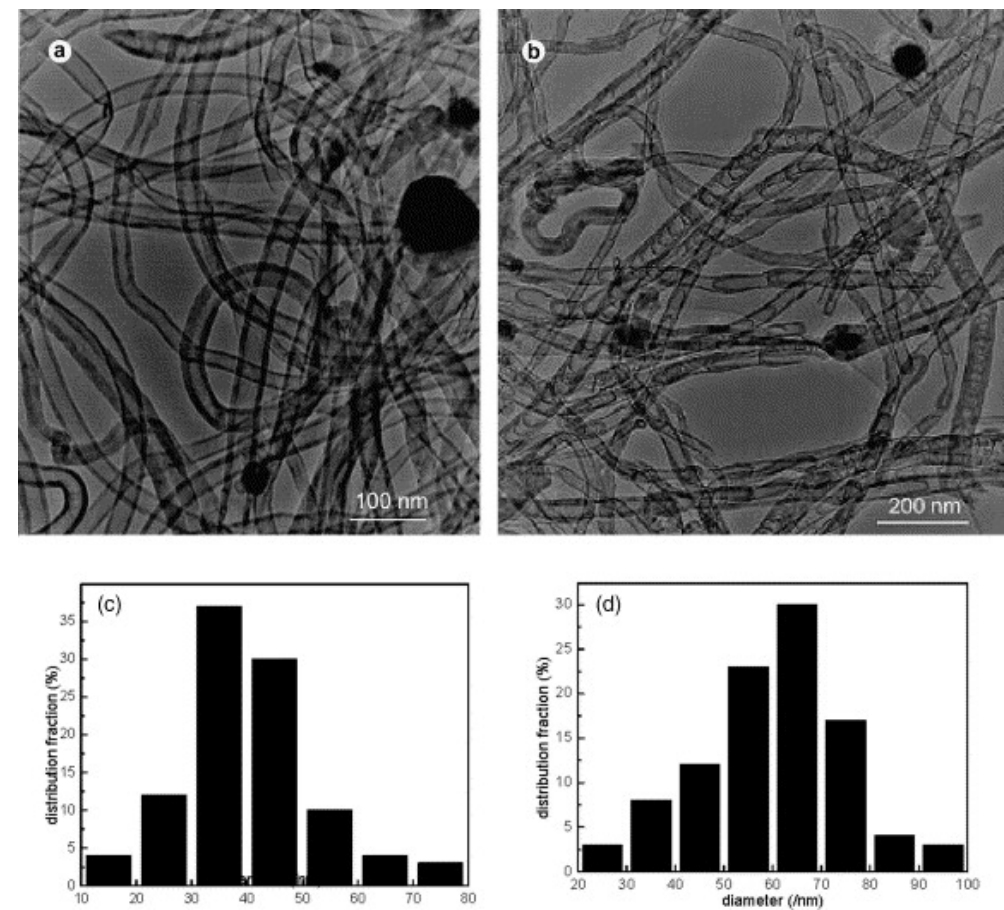

Fig. 1. (a) Typical TEM image of the product obtained from a detonation of $\mathrm{PA}-\mathrm{Co}(\mathrm{AC})_{2}$-paraffin with a mass ratio of 20:1:5 and PA loading density of $0.2 \mathrm{~g} / \mathrm{cm}^{3}$ (sample 1), showing that the grown tubes are dominant with normal CNTs with continuous hollow channels. (b) TEM image of the product obtained from a detonation of $\mathrm{PA}-\mathrm{Co}(\mathrm{AC})_{2}-$ paraffin with a mass ratio of $30: 3: 10$ and PA loading density of $0.2 \mathrm{~g} / \mathrm{cm}^{3}$ (sample 5), showing that most of the tubes exhibit bamboo shape. Statistical diameter distributions of the normal CNTs from sample 1 (c) and the bamboo-shape CNTs from sample 5 (d).

High paraffin/PA ratio is disadvantageous for the growth of normal tubes, but beneficial for the formation of bamboo-shape tubes (see samples 1-3 in Table 1). Since PA and $\mathrm{Co}(\mathrm{AC})_{2}$ contain high percent of oxygen atoms, which consume carbons as $\mathrm{CO}_{x}$ gases, they contribute relatively small fraction of the carbons to nanotube formation in the reaction system. Majority of the tube carbons come from the decomposition of paraffin. Paraffin/PA ratio actually determines the density of small carbon building blocks behind the decomposition reaction (Table 1) and thus the rate of the subsequent carbon deposition. The significant increase of $C_{\text {bamboo }} / C_{\text {normal }}$ ratio with increasing paraffin/PA ratio indicates that the formation of bambooshape tubes is related with high rate of carbon deposition. An overdose of paraffin (paraffin/PA $>1 / 2$ ) overburdens the catalyst nanoparticles and results in a dominant formation of amorphous carbon particles.

Comparing with the data of samples 2, 4-7 (Table 1), the formation of the bamboo-shape tubes seems also to be associated with relatively high $\mathrm{Co}(\mathrm{AC})_{2} / \mathrm{PA}$ ratio. In the present system, $\mathrm{Co}(\mathrm{AC})_{2}$ serves as catalyst precursor and, during the reaction, is converted into nanosized metal particles, which catalyze and control CNT growth. The percentage of $\mathrm{Co}(\mathrm{AC})_{2}$ is closely related to the $\mathrm{C} / \mathrm{Co}$ atomic ratio and to the size of the formed catalyst particles. Excessive $\mathrm{Co}(\mathrm{AC})_{2}$ (for example, $\mathrm{Co}(\mathrm{AC})_{2} / \mathrm{PA}>1 / 3$ ) leads to the products dominant with large encapsulated cobalt particles other than nanotubes, likely due to the size increase of cobalt particles hindering the diffusion of carbon species within the particles, which was believed to be crucial in the catalytic growth of tubes [17]. Large catalyst particles may be responsible for the formation of the bamboo-shape tubes, as described below.

The optimal conditions for the formation of bamboo-shape tubes are typically at $\mathrm{Co}(\mathrm{AC})_{2} / \mathrm{PA}$ and paraffin/PA ratios of $1 / 10$ and $1 / 3$, respectively, and at a PA loading density of $0.2 \mathrm{~g} / \mathrm{cm}^{3}$. Under this condition, almost all of the formed CNTs are in bamboo shape (Fig. 1b). These bamboo-shape CNTs, constructed by many jointed hollow compartments, have diameters of 
20-100 nm (peaked at about $65 \mathrm{~nm}$ ), which are obviously larger than the diameters of normal nanotubes $(10-80 \mathrm{~nm}$, peaked at $35 \mathrm{~nm})$, as shown in Fig. 1c and d. The large diameters of bamboo-shape tubes likely result from the large sizes of cobalt catalyst particles since the high $\mathrm{Co}(\mathrm{AC})_{2} / \mathrm{PA}$ mass ratio (1/10) used, compared with the value used in the syntheses of the normal CNTs. TEM observations really revealed an increase in the sizes of the catalyst particles under the conditions. It has been widely accepted that the size of catalyst particles controls the diameters of the grown tubes [18].

\subsection{Morphologies and structures of bamboo-shape CNTs}

Careful observations of the bamboo-shape CNTs by TEM reveal that their morphologies are highly distinctive, although all of them are constructed by partitioned short hollow compartments. We categorize the bamboo-shape CNTs into two types. The first type, as displayed by typical TEM and HRTEM images (2a-d), is characterized that their hollow compartments are normally spaced by curved carbon domes with carbon sheets joining the tube inner walls (denoted as type I hereafter). The outermost graphene sheets are structured continuously along the tube length. The thickness of the inner dome walls is always thinner than that of the tube walls. This type of bamboo-like tubes is structurally similar to those frequently obtained by other methods [1], [2], [6], [7], [8], [9] and [10]. However, unlike the previous observations of arc-resulted tubes [1] and [2], the dimensions of the different
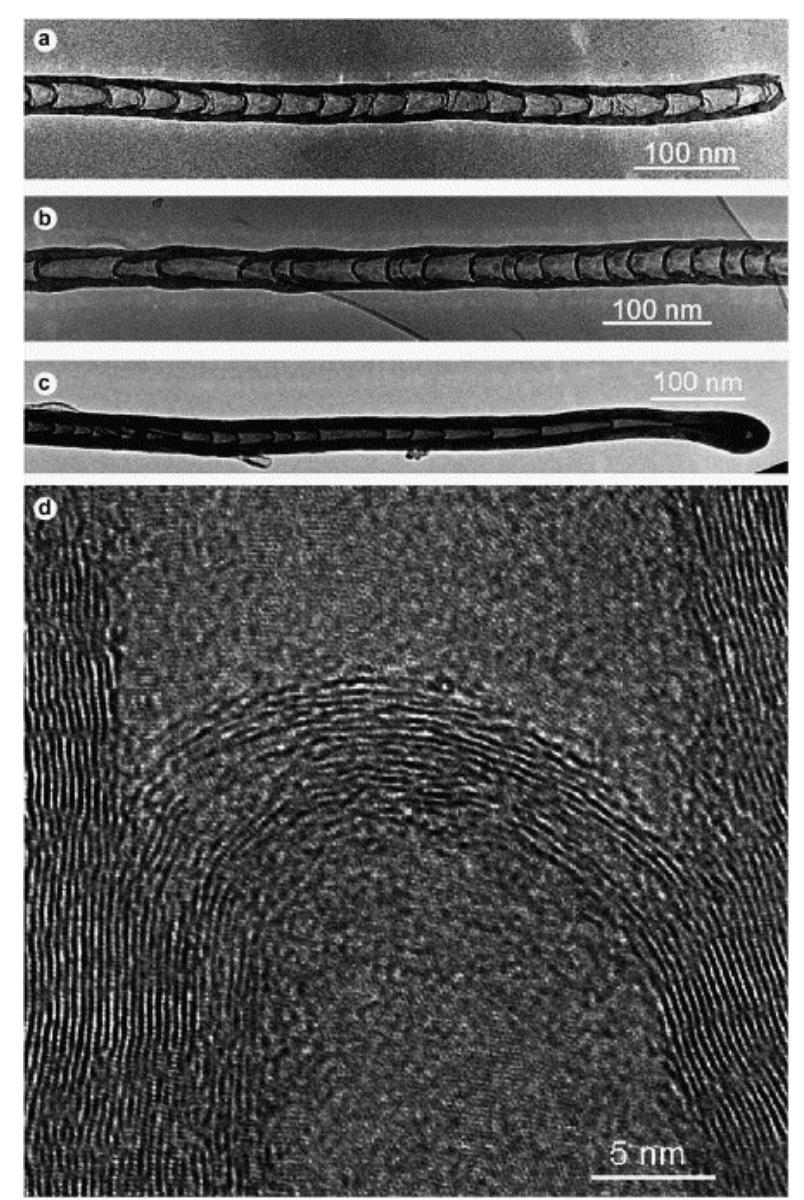
compartment cavities in an individual tube are not always uniform (Fig. 2a-c). Many tubes obtained by the present method exhibit compartment cavities with obviously different dimensions (Fig. 2b and c), which could be the consequence of a fluctuation in local growth environment. Another characteristic of this type of bamboo-shape tubes is that the compartment at one tube end normally contains a cone-shaped catalyst particle, but the middle compartments are empty, free from the catalyst particles (Fig. 2c), which is similar to the situation of normal tubes [14], [15] and [16].

Fig. 2. TEM images of the type I bamboo-shape nanotubes constructed by the compartments with uniform dimensions (a) and with different dimensions (b and c). (c) A conical catalyst particle is caged in the end compartment. (d) HRTEM image of a typical type I tube.

For the second type of bamboo-shape tubes, the compartments gather together by means of chaining, that is, the whole tube is constructed by connecting the open end of one compartment with the closed dome of another compartment (Fig. 3a-c, denoted as type II hereafter). This type of bamboo-shape nanotubes is rich in our samples, especially in those produced at high $\operatorname{Co}(\mathrm{AC})_{2} / \mathrm{PA}$ ratio. Their diameter varies along the tube axis since the different compartments and the different parts of the compartment units show quite different 
dimensions and shapes. Each compartment exhibits a narrow neck nearby the closed dome and the diameter of the closed dome is significantly larger than that of the open side (Fig. 3ac). The wall thickness is also not constant along the compartment, declining smoothly from the closed dome to the open side. Such a structure is somewhat similar to the $\mathrm{CN}_{x}$ "nanobell"gathered fibres produced by plasma-enhanced CVD technique [11] and [12], although the individual nanobell is much shorter than the individual compartment of the type II tubes. Fig. 4a shows a typical HRTEM image, which illustrates the jointing mode between the compartments. At the junctions, the jointing graphene sheets are clearly not successive (arrows). At some situations, the walls of the open end joint together and thus leave behind a needle-like structure without hollow channel, which adheres to the closed dome of another compartment, as shown by low resolution TEM image (arrow in Fig. 3c) and HRTEM image (Fig. 4b). Different from type I, in this type, catalyst particles are not only filled in the compartments at the tube ends, but also frequently observed at the middle parts in many compartments (Fig. 3).
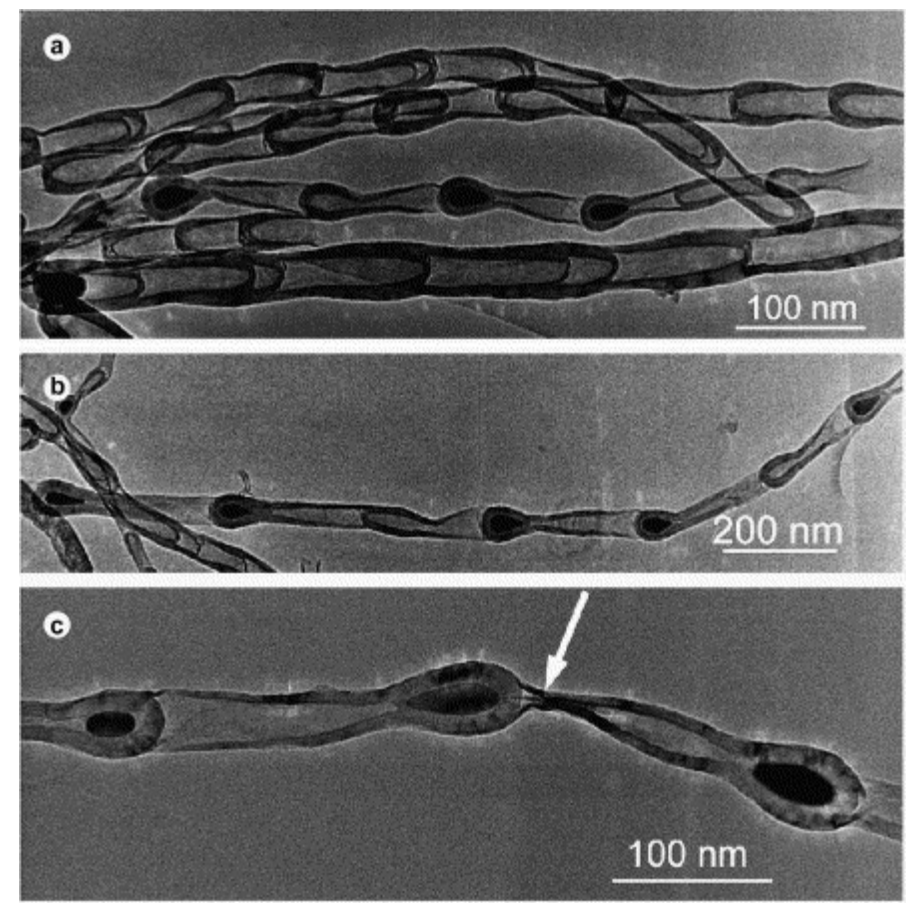

Fig. 3. TEM images of type II bamboo-shape nanotubes. Their compartment units have quite different diameters at different sections. Catalyst particles are not only filled in the compartments at the tube ends but also frequently caged in the compartments at the middle of the nanotubes.
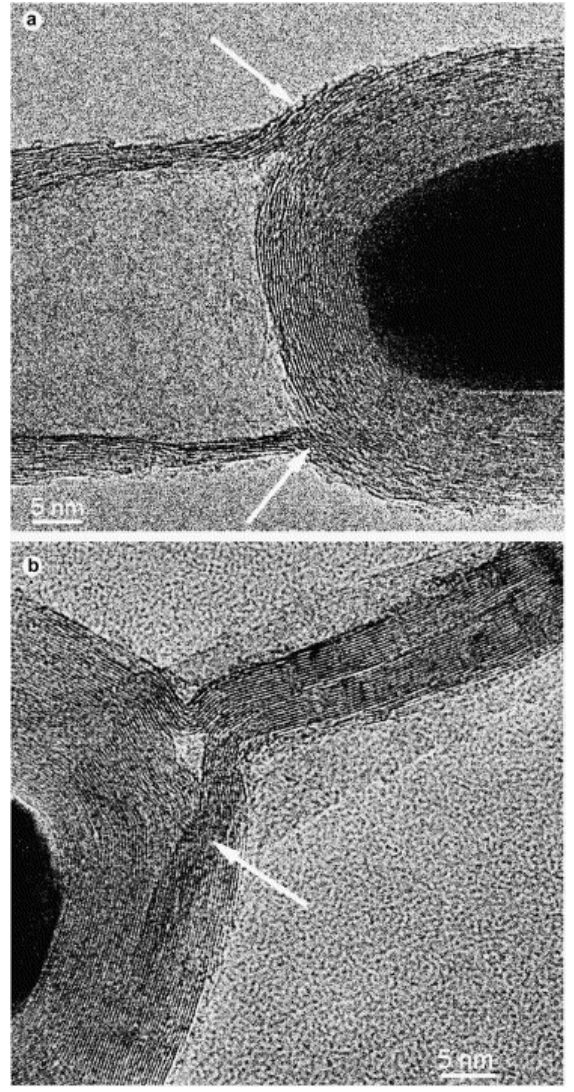

Fig. 4. HRTEM images of the junctions of the type II tubes. The arrows point the discontinued graphene sheets at the joint of two compartments.

\subsection{The growth of bamboo-shape CNTs}

For the normal CNTs, it is generally accepted that the catalytic growth process consists mainly of three steps: small carbon species adsorb, dissolve, and diffuse in catalyst particles, then graphene layers nucleate on the surfaces of the catalyst particle, and finally grow into tubular structures [19], [20] and [21]. This mechanism can well explain the growth process of the normal tubes formed in the detonation approach [15]. 
Fig. 5 shows an interesting tube structure. It is constructed by half of a normal tube with a long hollow channel and half of a bamboo-shape tube with partitioned hollow compartments. Such hybrid tubes possibly result from a perturbation of local physical and chemical environment and suggest that the formation mechanism of bamboo-shape tubes is closely correlative with that of normal tubes. XRD analysis (Fig. 6, sample 5) reveals that the catalyst particles formed under the conditions favorable to the growth of bamboo-shape tubes have a face-centered cubic structure of parent cobalt, the same as that of the catalyst particles produced under the conditions favorable to the growth of normal tubes [15]. This suggests that the formation of bamboo-shape tubes is not controlled by the catalyst crystalline structure.

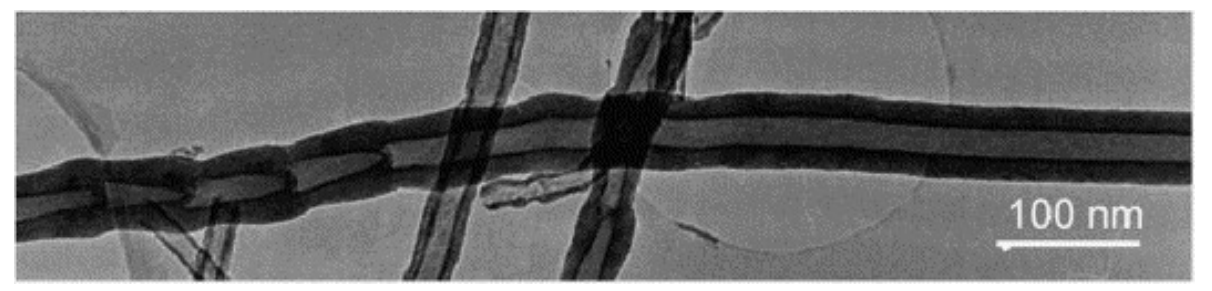

Fig. 5. TEM image of a special tube (the thwart one) constructed by half of a normal tube with a long hollow channel and half of a bamboo-shape tube with partitioned small hollow compartments.

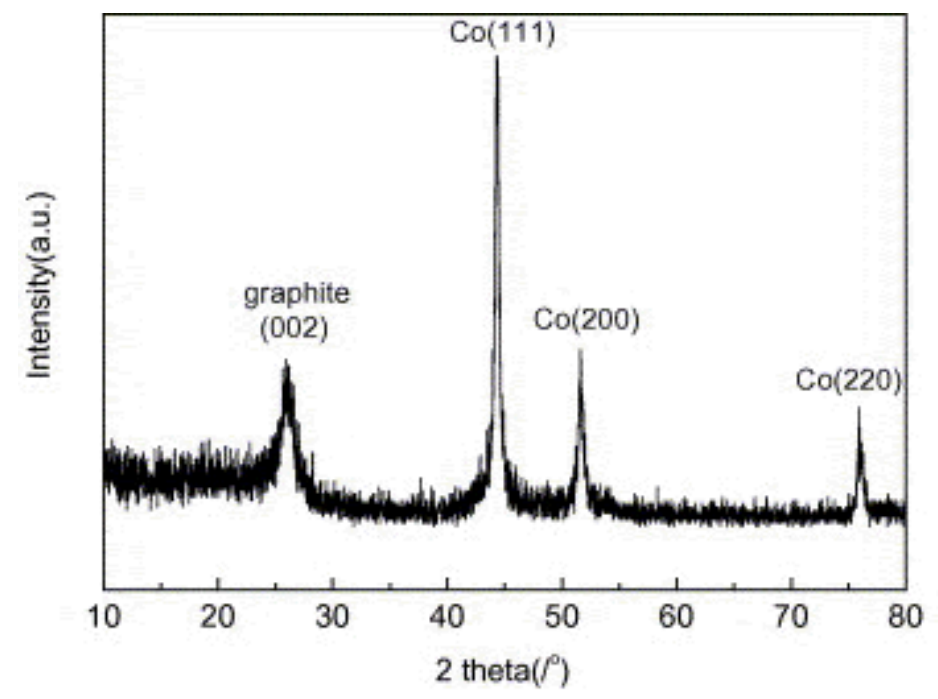

Fig. 6. XRD pattern of the sample 5.

Saito et al. [1] and [2] proposed that bamboo-shape tubes are formed from "jumps" of the metal particles out of the graphite sheath to the top of the tube at regular time intervals, driven by the stress accumulated in the graphitic sheath. Kovalevski and Safronov [6] further suggested that the bamboo-shape tubes are formed when the moving rate of the catalyst particle is slightly slower than the growing rate of the graphitic shell. These proposals, based on a conception that the growth of a tube is catalyzed by single catalyst particle, can explain the formation of the type I bamboo-shape carbon nanotubes because, as mentioned above, only one catalyst particle exists at the end of this type of tubes. It should be pointed out that some authors found that the presence of nitrogen-containing gases $\left(\mathrm{N}_{2}\right.$ or $\left.\mathrm{NH}_{3}\right)$ promotes the formation of bamboo-shape tubes [11], [12], [13], [22], [23] and [24], but our experimental results show that $\mathrm{N}_{2}$ does not play a key role in their formation, at least in our reaction system. Although a large amount of $\mathrm{N}_{2}$ is produced from the decomposition of PA and involved during the tube growth, normal tubes can be obtained under a similar $\mathrm{N}_{2}$-rich environment by controlling other experimental parameters. 
The difference in morphologies and structures between the two types suggests that the growth of the type II nanotubes may follow a different mechanism. The phenomenon that many catalyst particles exist in different compartments (Fig. 3) suggests that the growth of this type of tubes is catalyzed by more than one catalyst particles. The discontinuity of the graphene sheets at the joints between two compartments (Fig. 4) indicates that the type II bambooshape nanotubes are not grown continuously. The compartments seem to be welded together. These suggestions are also supported by the observations of some special appearances of this type of tubes. Fig. 7a displays a whip-like bamboo-shape tube, whose diameter changes not only within single compartment but also gradually from one end to the other end along the whole tube, and the thick end is about two times larger than the thin end. At the curved end of each compartment, there is a catalyst particle. Clearly, the formation of such a tube is not catalyzed by one cobalt particle. Fig. 7b shows another type II nanotube, of which the growth is directionally inversed, suggesting a discontinuous growth process of the tube.
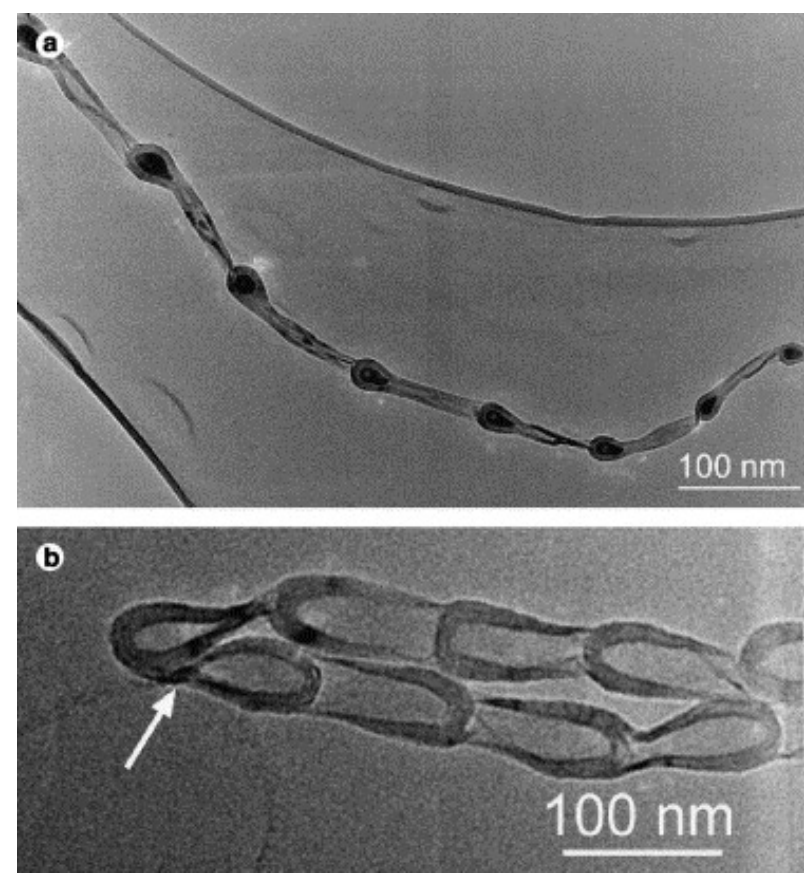

Fig. 7. (a) A whip-like tube, in which diameter changes not only in single compartment but also gradually from one end to the other end along the whole tube. (b) A highly winded type II tubes, of which the growth is directionally inversed at the joint of two compartments marked by the arrow.

The type II nanotubes likely result from a non-equilibrium growth-termination-growth process. In an individual type II bamboo-shape nanotube, each compartment is morphologically much similar to a short tube with an open end, and the existed catalyst particle is completely wrapped at the closed end of the compartment (Fig. 3). One can imagine that the compartments grow following the same mechanism as normal tubes, and the growth termination occurs as the catalyst particles are wrapped by graphene shells completely [25], [26], [27] and [28]. Wang and co-workers [12] have discussed the growth and formation of individual $\mathrm{CN}_{x}$ nanobell, which is morphologically analogous to some compartment units of the type II nanotubes. They speculated that the N-containing five-member rings, which result from the $\mathrm{N}$-doping-induced $\mathrm{sp}^{3}$ configuration of $\mathrm{C}-\mathrm{N}$ bonds, lead to the formation of conical structure of nanobell unit since the presence of pentagons creates a positive curvature. As revealed by the EELS elemental analyses (Fig. 8), the bamboo-shape nanotubes produced in the present technique are structured by pure carbon (containing no nitrogen element). It indicates that the presence of nitrogen element may be helpful but is not a critical factor to the formation of bamboo-shape nanotubes. The positive curvatures of their compartments could be also derived from pure-carbon pentagons, caused by the local perturbation of environments during nanotubes growing. 


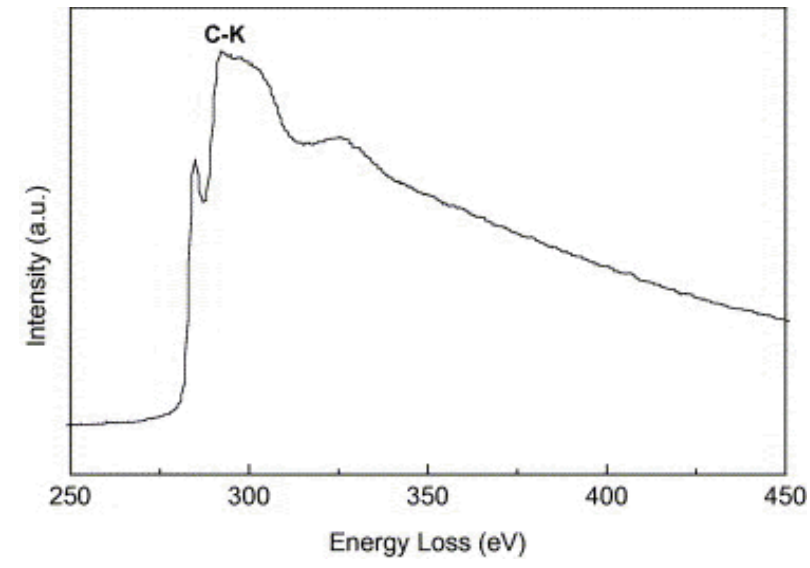

Fig. 8. EELS spectrum recorded from a highly curved dome of an individual compartment of bamboo-shape nanotube. It shows a peak at $\approx 290.9 \mathrm{eV}$, corresponding to the K-shell ionization edge of graphitic carbon. No nitrogen-characteristic peak was detected at $\approx 400 \mathrm{eV}$, indicating the curved dome was nitrogen free.

How the compartment units to form elongated bamboo-like tubes is of interest. In our system, the catalyst particles are uniformly dispersed in the whole space of the reactor after the rapid decomposition. A living catalyst particle can interact easily with the open edge of a terminated compartment and catalyze the formation of a new compartment. A repeating of this process leaves behind a long type II bamboo-shape nanotube. The open edge of each terminated compartment can accommodate only one living catalyst particle (possibly resulted from the structural stability of the open edge), which catalyzes the growth of another new compartment and thus leads to the formation of one-dimensional thread structure other than branched structure. Such a proposal is supported by the observations as follows. (a) In our experiments, the type II tubes are frequently formed when the ratio of $\operatorname{Co}(\mathrm{AC})_{2} / \mathrm{PA}$ is high. Such a condition results in formation of many large-sized catalyst particles, which may quicken the growth termination due to the difficulty of the carbon diffusion within the large particles (carbon-encapsulated catalyst particles, viewed as undeveloped tubes, were often formed under this condition) and simultaneously increase the chance of a terminated tube catching a new catalyst particle. In contrast, appropriately low mass ratio of $\mathrm{Co}(\mathrm{AC})_{2} / \mathrm{PA}$ favors the abundant formation of normal nanotubes. (b) High paraffin/PA ratio is also favorable for the formation of the type II bamboo-shape nanotubes. High paraffin/PA ratio induces high density of carbon species after the detonation and hence high carbon deposition rate, which likely also terminates the tube growth quickly. (c) This type of tubes is frequently winded, and the winding often occurs at the joints of two compartments with different winding degree (Fig. $3 \mathrm{~b}$ and c, Fig. $7 \mathrm{~b}$ ). On the other hand, although many compartments contain catalyst particles for the type II tubes, some compartments are empty. The real reason of such a phenomenon is not very clear, but is possible associated with an escaping [6], [29] and [30] of some catalyst particles that initially filled inside the compartments.

\section{Conclusion}

During the growth of carbon nanotubes in the rapid decomposition of the mixture of picric acid, acetate cobalt and paraffin, the morphologies of the formed tubes are highly sensitive to the loading density of picric acid and the mixture recipe. Morphologically pure normal tubes or bamboo-shape tubes can be selectively obtained by controlling the reaction conditions. Low PA loading density - associated with low system temperatures, high $\operatorname{Co}(\mathrm{AC})_{2} / \mathrm{PA}$ ratio - associated with large catalyst particles, and high paraffin/PA ratio-associated with high carbon species concentrations favor the formation of bamboo-shape tubes. 
The morphologies of the produced bamboo-shape tubes can be divided into two types. The graphene structures and the participation of the involved catalyst particles are also quite different between the two types of the tubes. Their growth follows different mechanisms. The formation of the type I tubes may follow a continuous growth mechanism determined by the rate of the movement of single catalyst particle and the rate of tube growth, as previously proposed by other researchers. The formation of the type II tubes is likely in a way characterized as a discontinued growth-termination-growth process, involving many catalyst particles for one tube. In addition, achieving morphologically pure bamboo-shape tubes make it possible to give insights into the chemical and physical properties of these specialstructured carbon nanotubes and to develop possible practical applications.

\section{Acknowledgments}

We thank National Natural Science Foundation of China for partial financial support (No. 59872047). This work was performed in frame of ELCASS. Z.P. Zhu thanks Alexander von Humboldt Foundation for fellowship support.

\section{References}

[1] Y. Saito and T. Yoshikawa, Bamboo-shape carbon tube filled partially with nickel, J. Cryst. Growth 134 (1993) (1-2), pp. 154-156

[2] Y. Saito, Nanoparticles and filled nanocapsules, Carbon 33 (1995) (7), pp. 979-988.

[3] B. Gan, J. Ahn, Q. Zhang, Rusli, S.F. Yoon and J. Yu et al., Y-junction carbon nanotubes grown by in situ evaporated copper catalyst, Chem. Phys. Lett. 333 (2001) (1-2), pp. 23-28.

[4] S. Subramoney, R.S. Ruoff, D.C. Lorents and R. Malhotra, Radial single-layer nanotubes, Nature 366 (1993) (6456), p. 637.

[5] Y. Wen and Z. Shen, Synthesis of regular coiled carbon nanotubes by Ni-catalyzed pyrolysis of acetylene and a growth mechanism analysis, Carbon 39 (2001) (15), pp. 23692374

[6] V.V. Kovalevski and A.N. Safronov, Pyrolysis of hollow carbons on melted catalyst, Carbon 36 (1998) (7-8), pp. 963-968.

[7] X. Wang, W. Hu, Y. Liu, C. Long, Y. Xu and S. Zhou et al., Bamboo-like carbon nanotubes produced by pyrolysis of iron(II) phthalocyanine, Carbon 39 (2001) (10), pp. $1533-1536$.

[8] C.J. Lee and J. Park, Growth model for bamboolike structured carbon nanotubes synthesized using thermal chemical vapor deposition, J. Phys. Chem. B 105 (2001) (12), pp. $2365-2368$.

[9] Y. Li, J. Chen, Y. Ma, J. Zhao, Y. Qin and L. Chang, Formation of bamboo-like nanocarbon and evidence for the quasi-liquid state of nanosized metal particles at moderate temperatures, Chem. Commun. 12 (1999), pp. 1141-1142. 
[10] H. Murakami, M. Hirakawa, C. Tanaka and H. Yamakawa, Field emission from wellaligned, patterned, carbon nanotubes emitters, Appl. Phys. Lett. 76 (2000) (13), pp. 17761778 .

[11] X. Ma, E. Wang, W. Zhou, D.A. Jefferson, J. Chen and S. Deng et al., Polymerized carbon nanobells and their field-emission properties, Appl. Phys. Lett. 75 (1999) (20), pp. 3105-3107.

[12] X. Ma, E.G. Wang, R.D. Tilley, D.A. Jefferson and W. Zhou, Size-controlled short nanobells: growth and formation mechanism, Appl. Phys. Lett. 77 (2000) (25), pp. 41364138 .

[13] X. Ma and E.G. Wang, $\mathrm{CN}_{\mathrm{x}} /$ carbon nanotube junctions synthesized by microwave chemical vapor deposition, Appl. Phys. Lett. 78 (2001) (7), pp. 978-980.

[14] Y. Lu, Z. Zhu, W. Wu and Z. Liu, Catalytic formation of carbon nanotubes during detonation of m-dinitrobenzene, Carbon 41 (2003) (1), pp. 194-198.

[15] Y. Lu, Z. Zhu, W. Wu and Z. Liu, Detonation chemistry of a CHNO explosive: catalytic assembling of carbon nanotubes at low pressure and temperature state, Chem. Commun. 22 (2002), pp. 2740-2741.

[16] Y. Lu, Z. Zhu, W. Wu and Z. Liu, Preparation of carbon nanotubes by catalysis-assisted detonation, Chem. J. Chin. U 24 (2003) (6), pp. 1063-1066.

[17] J.-W. Snoeck, G.F. Froment and M. Fowles, Filamentous carbon formation and gasification: thermodynamics, driving force, nucleation, and steady-state growth, J. Catal. 169 (1997) (1), pp. 240-249.

[18] Y.H. Tang, Y.F. Zheng, C.S. Lee, N. Wang, S.T. Lee and T.K. Sham, Carbon monoxideassisted growth of carbon nanotubes, Chem. Phys. Lett. 342 (2001) (3-4), pp. 259-264.

[19] C.J. Lee, J. Park, Y. Huh and J.Y. Lee, Temperature effect on the growth of carbon nanotubes using thermal chemical vapor deposition, Chem. Phys. Lett. 343 (2001) (1-2), pp. $33-38$.

[20] Z. Yao, H.W.C. Postma, L. Balents and C. Dekker, Carbon nanotube intramolecular junctions, Nature 402 (1999) (6759), pp. 273-276.

[21] B. Kitiyanan, W.E. Alvarez, J.H. Harwell and D.E. Resasco, Controlled production of single-wall carbon nanotubes by catalytic decomposition of $\mathrm{CO}$ on bimetallic $\mathrm{Co}-\mathrm{Mo}$ catalysts, Chem. Phys. Lett. 317 (2000) (3-5), pp. 497-503. |

[22] X.X. Zhang, Z.Q. Li, G.H. Wen, K.K. Fung, J. Chen and Y. Li, Microstructure and growth of bamboo-shape carbon nanotubes, Chem. Phys. Lett. 333 (2001) (6), pp. 509-514.

[23] C.J. Lee, S.C. Lyu, H.-W. Kim, J.H. Lee and K.I. Cho, Synthesis of bamboo-shape carbon-nitrogen nanotubes using $\mathrm{C}_{2} \mathrm{H}_{2}-\mathrm{NH}_{3}-\mathrm{Fe}(\mathrm{CO})_{5}$ system, Chem. Phys. Lett. 359 (2002) (1-2), pp. 115-120. 
[24] W.-Q. Han, P. Kohler-Redlich, T. Seeger, F. Ernst, M. Rühle and N. Grobert et al., Aligned $\mathrm{CN}_{\mathrm{x}}$ nanotubes by pyrolysis of ferrocene $/ \mathrm{C}_{60}$ under $\mathrm{NH}_{3}$ atmosphere, Appl. Phys. Lett. 77 (2000) (12), pp. 1807-1809.

[25] R.T.K. Baker, Catalytic growth of carbon filaments, Carbon 27 (1989) (3), pp. 315-323.

[26] S. Amelinckx, X.B. Zhang, D. Bernaerts, X.F. Zhang, V. Ivanov and J.B. Nagy, A formation mechanism for catalytically grown helix-shaped graphite nanotubes, Science $\mathbf{2 6 5}$ (1994) (5172), pp. 635-639.

[27] Smalley RE, Colbert DT. Self-assembly of fullerene tubes and balls. In: Robert A., editor. Welch foundation 39th conference on chemical research: nanophase chemistry, 1-10, Houston, 1995.

[28] J.-W. Snoeck, G.F. Froment and M. Fowles, Kinetic study of the carbon filament formation by methane cracking on a nickel catalyst, J. Catal. 169 (1997) (1), pp. 250-262.

[29] D. Ugarte, How to fill or empty a graphitic onion, Chem. Phys. Lett. 209 (1993) (1-2), pp. 99-103.

[30] H. Hou, A.K. Schaper, F. Weller and A. Greiner, Carbon nanotubes and spheres produced by modified ferrocene pyrolysis, Chem. Mater. 14 (2002) (9), pp. 3990-3994. 